\title{
Pityriasis rosea following COVID-19 flu vaccination
}

\section{Lorenzo Martini ${ }^{1,2}$}

\author{
${ }^{1}$ University of Siena, Department of Pharmaceutical Biotechnologies, Via A. Moro 2, 53100 Siena, Italy, ${ }^{2}$ C.R.I.S.M.A. Inter \\ University Centre for Researched Advanced Medical Systems, Via A. Moro 2, 53100 Siena, Italy
}

Corresponding author: Lorenzo Martini, MD, e-mail: lorenzo.martini@unisi.it

Sir,

Camille-Melchior's Pityriasis (alias Pityruasis rosea) is a distinct papulosquamous skin eruption that has been attributed to viral reactivation, certain drug exposures or rarely, vaccination.

This should be a clinicopathlogically typical case of pityriasis rosea that developed after an anti-covid vaccination. With a global vaccination program against the pandemic flu, patients should be apprised of the possibility of such rare but benign skin reaction to avoid unnecessary fear. Pityriasis rosea (PR) is an acute, selflimited, papulosquamous skin eruption that occurs most commonly among teenagers and young adults [1]. Typical clinical presentation is the appearance of the primary herald patch followed by the onset of secondary scaly skin eruptions characteristically distributed along the skin tension line within days to weeks [2]. Although PR is a well-known and relatively common disease, its cause is still not completely understood. Here I intend to report a case of PR occurring after an anti-covid vaccination. This case may offer an example of possible skin adverse reaction associated with this "new vaccine" and in addition raise issues about the etiopathogenesis of vaccination-associated PR $[3,4]$.

The subject was a 25 -year-old male who presented a drastic skin rash on his trunk and proximal limbs which had been present for one entire week. The patient described that a herald skin lesion was spotted initially in his left upper thigh followed several days later by the onset of many itchy scaly lesions on his trunk and proximal extremities. The first herald patch developed five days after he underwent vaccination. There were no systemic symptoms such as fever, malaise, myalgia or loss of appetite. He had no history of egg allergies, recent infection or drug exposure. He also denied any personal or family history of similar skin eruptions. On physical examination, the herald patch on his left upper thigh showed a $3-\mathrm{cm}$ oval thin plaque with a central, wrinkled, erythematous area and a salmon-colored peripheral zone. The secondary eruptions were numerous, discrete, $1-3 \mathrm{~cm}$ in diameter, oval to round, salmon-colored plaques with similar collarette of scale that looked like the primary herald patch in miniature. The distribution of these secondary plaques was along the lines of cleavage that assumed a Christmas tree pattern on the patient's trunk.

We prayed him to make a skin biopsy obtained from one trunk lesion and the result demonstrated patchy parakeratosis, reduced granular cell layer, mild acanthosis and mild spongiosis. The superficial dermis revealed slight papillary edema, perivascular and superficial dermal interstitial infiltrate of lymphocytes and histiocytes. The diagnosis of PR was made because of the typical clinicopathologic features.

We have treated the volunteer with oral antihistamine and mid-potency topical steroid cream. At the scheduled 2-week follow-up visit, the itch symptoms as well as the skin lesions were improved.

Covid flu is a highly infectious respiratory disease that may potentially cause considerable morbidity and mortality in high risk populations [4]. In effect We have discovered a top secret report that refers that some researchers in Taiwan had analyzed the data retrieved from the Taiwan Centers for Disease Control of suspected $\mathrm{HlNl}$ vaccine-related adverse events weekly summary reports database dated from November 1, 2009 to February 4, 2010 [5]. The total number of suspected adverse events attributed to $\mathrm{HlNl}$ vaccination was 1247. Among them, the skin adverse events comprised 260 cases, which was around $21 \%$ of the total reported adverse events (Table 1). The most commonly reported solicited adverse events in other

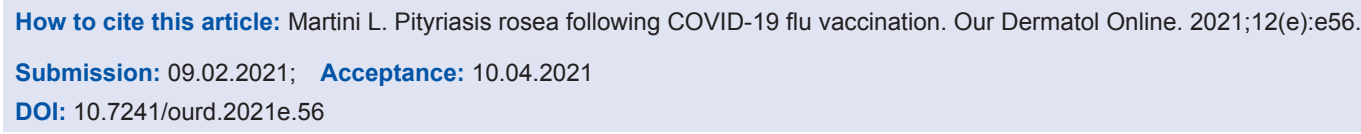


Table 1: Statistical data of suspected vaccine-related skin adverse events after $\mathrm{H} 1 \mathrm{~N} 1$ vaccination from Taiwan Centers for Disease Control dated from November 1, 2009 to February 4, 2010

\begin{tabular}{lcc}
\hline & $\begin{array}{c}\text { Number of } \\
\text { cases reported }\end{array}$ & $\begin{array}{c}\text { Percentage } \\
\text { (\%) }\end{array}$ \\
\hline Unspecified skin rash & 140 & 53.8 \\
Injection site reaction (reported as & 51 & 19.6 \\
erythema, swelling, induration, cyst, & & \\
mass formation, injection site pain, & & \\
pruritus and anesthesia) & 19 & 7.3 \\
Urticaria & 18 & 6.9 \\
Angioedema (including orbital, & & \\
auricular, lip, facial edema) & 5 & 1.9 \\
Unspecified pruritus & 4 & 1.5 \\
Mouth ulceration & 4 & 1.5 \\
Exfoliative rash & 4 & 1.5 \\
Purpura & 3 & 1.2 \\
Vasculitis & 2 & 0.77 \\
Cellulitis & 2 & 0.77 \\
Blister or vesicular rash & 2 & 0.77 \\
Herpes virus infection & 1 & 0.38 \\
Ecchymosis & 1 & 0.38 \\
Petechiae & 1 & 0.38 \\
Skin discoloration & 1 & 0.38 \\
Alopecia & 1 & 0.38 \\
Folliculitis & 1 & 0.38 \\
Unspecified dermatitis & 260 & - \\
Total number of cases reported & & \\
\hline
\end{tabular}

clinical trials were injection site reaction such as pain or erythema [6]. Unlike the data reported from other countries, the most reported adverse skin reaction in Taiwan was unspecified skin rash, comprising 53.8\%, followed by injection site reaction, which reached $19.6 \%$. The lower incidence of injection site reaction in Taiwan may result from the underreporting of these minor events. The incidence of both urticaria and angioedema was approximately $14 \%$. The remaining skin adverse events were the minority, including unspecified pruritus, mouth ulceration, exfoliative rash, vasculitis, cellulitis, blister or vesicular rash, herpes infection, ecchymosis, petechiae, skin discoloration, alopecia, folliculitis and unspecified dermatitis. No serious adverse skin reactions such as Stevens-Johnson syndrome or toxic epidermal necrolysis had been reported so far [7].

We can refer that approximatively the fate of people who underwent to covid vaccinations could present an avalanche of adverse symptoms, but the most impressive is the Pityriasis rosea that lasts almost 2 weeks.

\section{Consent}

The examination of the patient was conducted according to the principles of the Declaration of Helsinki.

The authors certify that they have obtained all appropriate patient consent forms, in which the patients gave their consent for images and other clinical information to be included in the journal. The patients understand that their names and initials will not be published and due effort will be made to conceal their identity, but that anonymity cannot be guaranteed.

\section{REFERENCES}

1. Brzezinski P, Sinjab AT. Pityriasis rosea in 12-months-old infant. Our Dermatology Online. 2012;2:119-22.

2. Chiriac A, Brzezinski P, Podoleanu C, Stolnicu S. atypical case of pityriasis rosea in a child following streptococcal erythema nodosum. J Interdiscip Med. 2017;2:354-6.

3. Brzezinski P, Chiriac A. Uncommon presentation of pityriasis rosea after yellow fever inoculation. JAMA Dermatol. 2014;150:1020-1.

4. Soundarya s, Sundaramoorthy S. Unmasking the mask: COVID-19 manifestations of PPE kits. Our Dermatol Online. 2020;11:e186.

5. Drago F, Broccolo F, Rebora A:Pityriasis rosea: an update with a critical appraisal of its possible herpesviral etiology: J Am Acad Dermatol, 2009;61:303-18.

6. Drago F, Rebora A. Viral reactivation and skin eruptions. Dermatology, 2003;207:1-2.

7. Gupta M. Pattern of cutaneous infections in pediatric age group - A clinico-observational study. Our Dermatol Online. 2020;11:35-7.

Copyright by Lorenzo Martini. This is an open access article distributed under the terms of the Creative Commons Attribution License, which permits unrestricted use, distribution, and reproduction in any medium, provided the original author and source are credited.

Source of Support: Nil, Conflict of Interest: None declared. 